\title{
Evaluation of Functional Status in Patients after Total Knee Arthroplasty: A Comparison of the Journey II Bi-Cruciate Stabilized Total Knee System and Genesis II Cruciate-Retaining Implant
}

\author{
Maciej Biały ${ }^{1,2}$, Ronny De Corte ${ }^{3}$, Juliusz Dec ${ }^{1}$, Adam Pierzchała ${ }^{1}$, Rafał Gnat ${ }^{2}$ \\ ${ }^{1}$ Functional Diagnostics Laboratory, Sport-Klinika, Poland \\ ${ }^{2}$ Institute of Physiotherapy and Health Sciences, Academy of Physical Education in Katowice, Poland \\ ${ }^{3}$ Knee Revision, Smith \& Nephew, Belgium
}

Correspondence to: Maciej Biały, email: mbfizjoterapia@gmail.com

DOI: https://doi.org/10.5114/phr.2021.109028

Received: 20.04.2021 Reviewed: 24.04.2021 Accepted: 24.04.2021

\begin{abstract}
Background: Total knee arthroplasty (TKA) is an effective treatment for degenerative knee osteoarthritis. The development of new implant designs not only focuses on pain relief but also aims to improve knee function after TKA.
\end{abstract}

Aims: The aim of this study was to evaluate and compare early functional outcomes after TKA using two different types of prosthesis.

Material and methods: Patients who underwent TKA with the Journey II Bi-cruciate Stabilized (JII BCS) Total Knee System $(\mathrm{n}=9)$ and Genesis II Cruciate-Retaining (GII CR) implant $(\mathrm{n}=9)$ were included in the functional evaluation. Active range of motion (AROM) was measured. Anterior tibial translation was measured using an electromechanical arthrometer, while single-leg stance performance was assessed using a balance platform. The Western Ontario and McMaster Universities Osteoarthritis (WOMAC) questionnaire was used for the assessment of subjective functional outcomes.
Results: The arthrometry result showed a significant difference of $(-) 0.2 \mathrm{~mm}$ between the operated and non-operated extremities in the JII BCS group and $2.3 \mathrm{~mm}$ in the GII CR group. The single-leg balance test result showed a significant difference in overall stability index of $2.7^{\circ}$ for the JII BCS group and $4.6^{\circ}$ for the GII CR group. Both the AROM and WOMAC scores were higher in the JII BCS group.

Conclusions: Compared with the GII CR implant, the JII BCS implant provided better functional outcomes of the anterior and posterior cruciate ligaments in patients in the early phase after TKA.

\author{
Key words \\ total knee arthroplasty, functional status, \\ Journey II Bi-cruciate Stabilized, \\ Genesis II Cruciate Retaining
}




\section{Introduction}

Total knee arthroplasty (TKA) is accepted as the gold standard for the treatment of severe knee osteoarthritis. While the expectations of patients are high [1], several studies have shown poorer functional outcomes after TKA [2,3] than after hip arthroplasty [4]. Noble et al. [5] provided objective information regarding the functional capacity of control subjects and patients who underwent TKA. They demonstrated that the patients who underwent TKA experienced substantial functional impairment compared with their ageand sex-matched peers, especially when performing biomechanically demanding activities [5]. They suggested that significant improvements in the procedure and prosthetic designs are urgently needed to restore the normal knee function after TKA.

Other studies that examined the outcomes of TKA using conventional implants reported abnormalities in knee joint kinematics, such as paradoxical anterior translation of the femur with respect to the tibia during knee flexion, insufficient or reverse axial rotation of the tibiofemoral joint, abnormal lateral position of the pivot point, and reduction in quadriceps efficiency [6-8]. In all these studies, TKA did not achieve successful restoration of the anterior cruciate ligament (ACL) function and thus resulted in ACL deficiency. Similar results were found in the work of Pritchett [9], who claimed that ACL deficiency after TKA causes paradoxical motion, which might be related to limitations in knee range of motion, reduction in quadriceps efficiency, and absence of the "feeling of a normal knee." Attempts have been made to replace the ACL function with newer implants designed to restore normal knee joint kinematics and stability. Recently, Murakami et al. [10] used an image-matching technique to measure in vivo knee joint kinematics during squatting and stair-climbing activities, and showed a reproducible and stable tibiofemoral translation during these motions, most likely caused by the morphological characteristics of the prosthesis designed to support the ACL function. In another study that investigated in vivo kinematics during gait, the
Journey II Bi-Cruciate-Stabilized (JII BCS) Total Knee System proved to be effective for restoring dynamic stability during gait compared with the posterior-stabilized implant. This can be explained by the JII BCS special design, which consists of anterior cam and weight-bearing surfaces and is aimed at mimicking the native ACL function and physiological shape of the knee joint line and articular surfaces [11]. Ries [12] compared the effect of ACL sacrifice, retention, or substitution on knee joint kinematics after TKA and suggested that the ACL function is necessary to achieve a more normal kinematics after TKA. When restoring the ACL function by means of prosthetic design and technology, two important features may influence the stability and kinematics of the knee joints after TKA, namely the geometry of the weight-bearing surfaces and the mechanical interaction between the polyethylene and femoral components, or the cam-post mechanism. The JII BCS TKA implant used in this study replicates the ACL and posterior cruciate ligament. The sagittal shape of the medial insert compartment conforms with the sulcus in the middle of the insert, while the lateral side is slightly convex. In addition, an anterior and asymmetrical posterior post-cam system prevents the tibia from moving too far forward or backward underneath the femur $[13,14]$.

\section{Aims}

This study aimed to verify the functional status of the lower extremity after TKA using the JII BCS Total Knee System compared with that using the Genesis II Cruciate-Retaining (GII CR) prosthesis.

\section{Materials and Methods}

Data were prospectively collected from two groups of patients who underwent TKA surgery with different types of prosthesis (JII BCS vs GII CR). A data set comprising of active range of motion (AROM), range of anterior tibial translation (ATT), single-leg balance test (SLBT) result, and functional score (WOMAC) was recorded in the early po- 
stoperative phase (between 3 and 4 months after TKA). All the subjects provided informed consent. A total of 18 patients who underwent primary TKA using the JII BCS (Smith \& Nephew, Memphis, USA) or GII CR implant (Smith \& Nephew) were evaluated in this study. Patients who met the following inclusion criteria were enrolled: age between 50 and 70 years, no history of previous knee surgery, and no additional surgery or current hip or ankle joint pain (for both lower extre- mities). After surgery each patient received supervised rehabilitation (by a physiotherapist not associated with this study) and precise guidelines for TKA management in the early postoperative phase, which were focused on knee range of motion, edema management, muscle strength drills, and exercise progression regimes. Both groups were matched for age, weight, height, and time interval from TKA to assessment (Table 1).

Table 1. Demographic characteristics of subjects in Journey II BCS and Genesis II CR groups, with no significant differences in all presented variables.

\begin{tabular}{|l|l|l|}
\hline Variable & JII BCS (N=9) & GII CR (N=9) \\
\hline Male/female ratio & $5: 4$ & 4.5 \\
\hline${\text { Patient age }(\mathrm{y})^{\mathrm{a}}}^{\mathrm{H}}$ & $60.8 \pm 6.5$ & $56.7 \pm 3.6$ \\
\hline Height $(\mathrm{m})^{\mathrm{a}}$ & $171.2 \pm 8.1$ & $170.4 \pm 6.3$ \\
\hline Weight $(\mathrm{kg})^{\mathrm{a}}$ & $82.3 \pm 7.4$ & $83.0 \pm 8.8$ \\
\hline Body mass index $(\mathrm{kg} / \mathrm{m} 2)^{\mathrm{a}}$ & $30.3 \pm 4.7$ & $32.1 \pm 2.7$ \\
\hline $\begin{array}{l}\text { Time interval from TKA } \\
\text { to assessment (months) }\end{array}$ & $3.4 \pm 0.5$ & $3.4 \pm 0.5$ \\
\hline
\end{tabular}

Notes: a Values expressed as mean \pm standard deviation.

Abbreviations: JII BCS - Journey II Bi-cruciate Stabilized Total Knee System; GII CR - Genesis II CruciateRetaining; TKA - total knee replacement.

A single rater, who was an experienced certified physiotherapist with 10 years of experience in orthopedic rehabilitation and 5 years of training in functional testing, was involved in the collection of the outcome data. A pilot study was conducted with 12 participants to achieve reliable levels of measurement for AROM, ATT, and singe-leg balance.

Knee AROM was measured in a supine position by using a long-arm goniometer, with the subject's arms aligned with the greater trochanter and lateral malleolus. The goniometer axis was placed in the center of the lateral femur condyle. For knee flexion, the patients were asked to flex the knee as far as possible by sliding the heel on a supporting surface. For knee extension, the heel was placed on a raised block, and the participant actively extended the knee [15]. All the subjects performed three repetitions in each direction of movement, and the mean value of the three measurements was used in the analysis. The reliability of knee ROM measurements was previously reported by Rothstein et al. [16]. In addition, intra-rater reliability was verified in a pilot study that showed a good level of intraclass correlation coefficient (ICC) for both flexion and extension AROM (both $\mathrm{ICC}_{3,3}=0.85$ ). ICC values $<0.5$, between 0.5 and 0.75 , between 0.75 and 0.9 , and $>0.90$ are indicative of poor, moderate, good, and excellent reliability, respectively [17]. 
For the ATT measurement, the GNRB arthrometer (Genourob, Laval, France), which performs an automated Lachman test, was utilized. The patient lay supine on the therapeutic table, with the body in line with the measurement device. In accordance with the manufacturer's instructions, the knee was placed in $20^{\circ}$ flexion in a molded support, with the joint line between the thigh and calf support, to reproduce the Lachman test position, following the description by Robert et al. [18]. The patella and foot of the tested leg were stabilized with the straps of the GRNB device. In the next step, a displacement sensor was positioned on the tibial tuberosity, and the upper part of the displacement sensor rod was positioned between 1 and $2.5 \mathrm{~cm}$ (Figure 1A). A force of up to $150 \mathrm{~N}$ was applied to the upper part of the calf by using the GRNB tensor in an anterior direction. For both the operated and non-operated extremities, three repetitions of ATT were recorded using the GRNB software. In the pilot study, the intra-rater reliability of the three repeated measurements was good $\left(\mathrm{ICC}_{3,3}=0.85\right)$.
The SLBT was performed using the Biodex Balance System (Biodex Medical Systems, Shirley, NY, USA), which utilizes sensors under its platform to detect postural sway. We performed the postural stability test to assess the patient's ability to maintain the center of balance on a platform, locked in a horizontal position. The Biodex Balance System measures the deviation of the patient's center of gravity from the center of the platform. SLBT includes three types of output measures, namely the overall (OA), anteroposterior (AP), and mediolateral (ML) stability indexes. These indexes show the standard deviations of the fluctuations around the reference point, which is a locked platform at the horizontal position, and are calculated by measuring the times during which the platform deviated from the horizontal position, along with the degree of angulation from the reference point. A low score is more desirable than a high score. The formulae for calculating OA, AP, and ML were previously described by Arnold and Schmitz [19]. All the participants were instructed to assume a single-leg stance on the firm plat-
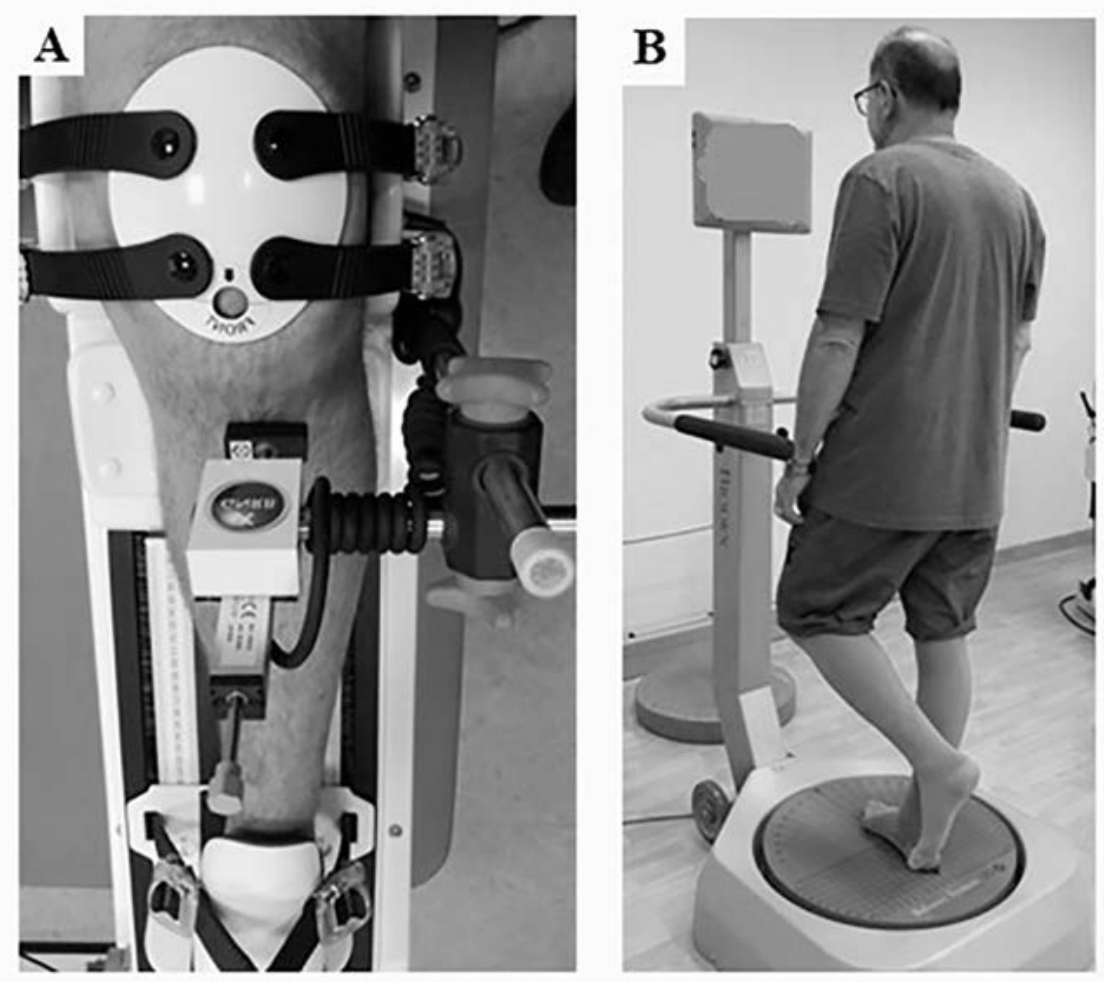

Figure 1. (A) Lower extremity alignment during anterior tibia translation measurement with GRNB arthrometer, and (B) patient position during single leg balance test, all measurements were taken without visual feedback. 
form, with the contralateral extremity flexed $90^{\circ}$ at the knee joint (Figure 1B). Each subject performed 3 repetitions of single-leg stance on the operated extremity for $10 \mathrm{~s}$ without visual feedback. The Biodex Balance System measurement was considered reliable [19]. The $\mathrm{ICC}_{3,3}$ values in the pilot study ranged from 0.80 to 0.88 .

The Western Ontario and McMaster Universities Osteoarthritis Index (WOMAC) is a self-administered questionnaire developed to study patients with hip and knee osteoarthritis. The final score was determined by adding the subsequent scores for pain, stiffness, and physical function. The questionnaire score ranges from 0 to 100 points, where 100 represents the worst possible health status. The WOMAC is reliable, valid, and sensitive to the changes in patients with knee osteoarthritis [20].

Differences in all the measured variables for the operated extremities were compared between the JII BCS and GII CR groups by using a non-parametric Mann-Whitney $U$ test. The alpha level was set at 0.05. The Statistica software (Statistica, Tulsa, USA) was used for all the calculations.

\section{Results}

The individual results of the measurement of AROM (flexion and extension) for the operated extremities are presented in Table 2, where (-) indicates hyperextension. The mean knee flexion angles achieved by the JII BCS and GII CR groups were $115.1^{\circ} \pm 13.8^{\circ}$ (range: $87^{\circ}-130^{\circ}$ ) and $102.5^{\circ}$ $\pm 11.2^{\circ}$ (range: $87^{\circ}-120^{\circ}$ ), respectively. The mean extension rates in the JII BCS and GII CR groups were $0.2^{\circ} \pm 2.2^{\circ}$ (range: $-3^{\circ}$ to $5^{\circ}$ ) and $6.6^{\circ} \pm$ $4.2^{\circ}$ (range: $0^{\circ}-12^{\circ}$ ), respectively. The differences in flexion and extension AROM between the two groups were not statistically significant $(p>0.05)$. When comparing the total AROM (flexion angle minus the extension angle), the mean range was $114.9^{\circ} \pm 15.6^{\circ}$ (range: $87^{\circ}-130^{\circ}$ ) in the JII BCS group and $95.9^{\circ} \pm 14.9^{\circ}$ (range: $98^{\circ}-120^{\circ}$ ) in GII CR group. The difference in total AROM between the two groups was statistically significant $(p=0.022)$.

Table 2. Active range of motion results for individual participants (operated extremity).

\begin{tabular}{|l|l|l|l|l|}
\hline Patient No. & JII BCS & GII CR & Extension \\
\hline & Flexion & Extension & Flexion & 11 \\
\hline 1 & 115 & -3 & 92 & 7 \\
\hline 2 & 98 & 2 & 112 & 6 \\
\hline 3 & 122 & 0 & 105 & 12 \\
\hline 4 & 87 & 5 & 90 & 8 \\
\hline 5 & 130 & 0 & 87 & 9 \\
\hline 6 & 117 & -2 & 101 & 6 \\
\hline 7 & 123 & 0 & 103 & 0 \\
\hline 8 & 118 & 0 & 112 & 0 \\
\hline 9 & 126 & 0 & 120 & 6.6 \\
\hline Mean 1-9 & 115.1 & 0.22 & 102.4 & 4.2 \\
\hline SD & 13.9 & 2.2 & 11.2 & $0-12$ \\
\hline Min-max & $87-130$ & $-3-5$ & $87-120$ & \\
\hline
\end{tabular}

Abbreviations: JII BCS - Journey II Bi-cruciate Stabilized Total Knee System; GII CR - Genesis II Cruciate-Retaining; $\mathrm{SD}$ - standard deviation; (-) - hyperextension. 
The patients in the JII BCS and GII CR groups had mean ATT values of $6.2 \pm 1.3$ and $8.3 \pm 3.5 \mathrm{~mm}$ for the operated extremity, respectively (Figure 2, left). The mean differences between the operated and non-operated extremities in the JII BCS and GII CR groups were -0.2 and $2.3 \mathrm{~mm}$, respectively (Figure 2, right).

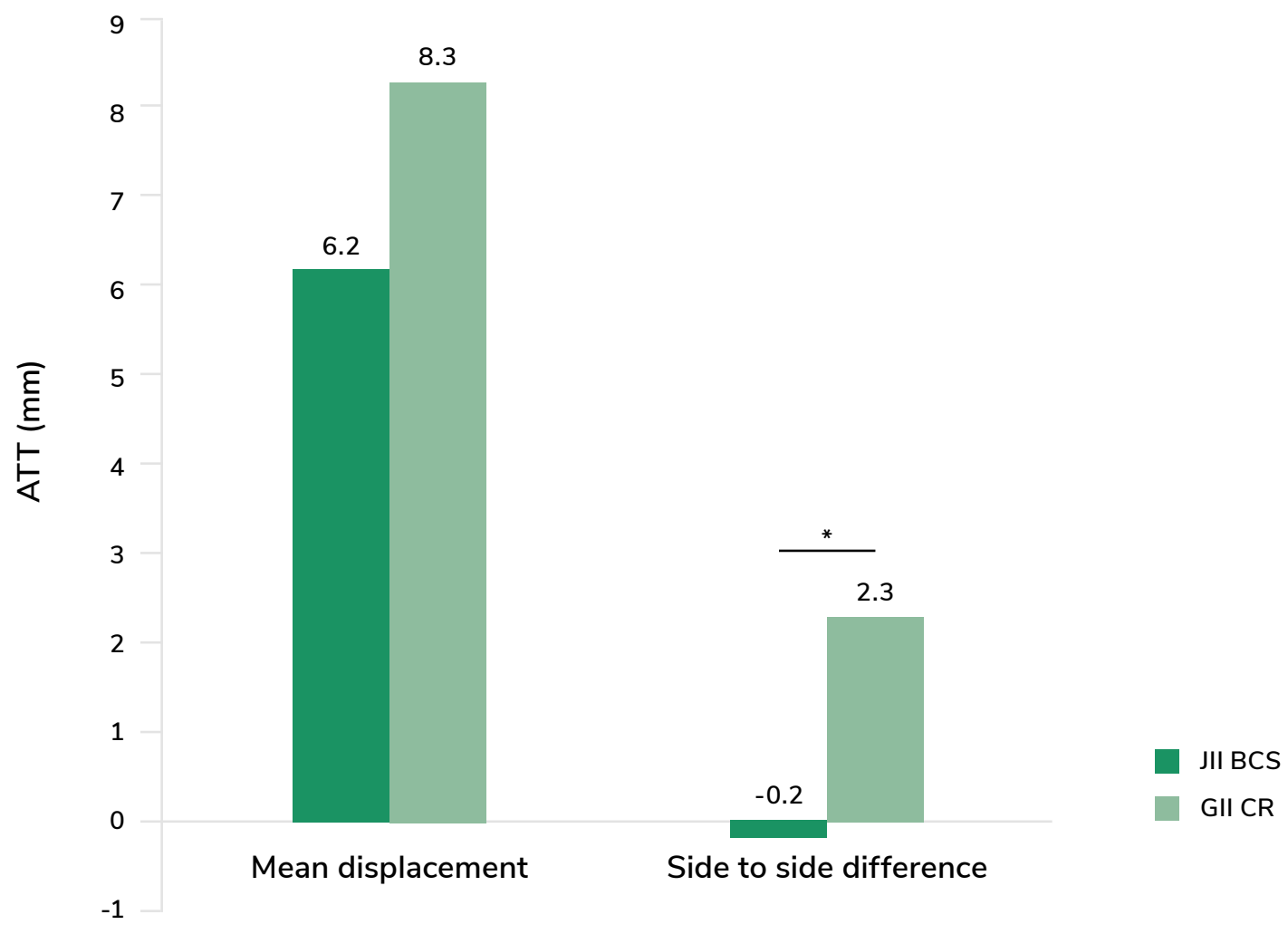

Figure 2. GNRB® arthrometry results in Journey II BCS (JII BCS) and Genesis II CR (GII CR) group; (ATT) anterior tibia translation; $\left({ }^{*}\right)$ statistically significant $(p<0.05)$.

Abbreviations: ATT - anterior tibia translation; JII BCS - Journey II Bi-cruciate Stabilized Total Knee System; GII CR - Genesis II Cruciate-Retaining.

When comparing the operated and non-operated extremities, we found a statistically significant difference between the groups $(p=0.00001)$. With the GNRB arthrometer, we measured the range of ATT and obtained information on the slope of the translation curves. In the JII BCS group, the mean difference in ATT between the operated and non-operated extremities was small $(0.2 \mathrm{~mm})$. This proves that the subjects had two stable knees with a slight side-to-side difference in ATT. However, in the GII CR group, we noticed visible signs of an increasing side-to-side difference in ATT, which correlated with the increase in the force applied to the knees.

The OA, AP, and ML stability index values were, respectively, $2.7^{\circ} \pm 1.5^{\circ}, 1.7^{\circ} \pm 0.8^{\circ}$, and $1.9^{\circ} \pm 1.6^{\circ}$ in the JII BCS group and $4.6^{\circ} \pm 1.4^{\circ}, 4.1^{\circ} \pm 1.3^{\circ}$, and $1.7^{\circ}$ $\pm 0.8^{\circ}$ in the GII CR (Figure 3 ). The differences in the OA and AP stability index values between the JII BCS and GII CR groups were statistically significant ( $p=0.0192$ and $p=0.0009$, respectively). 


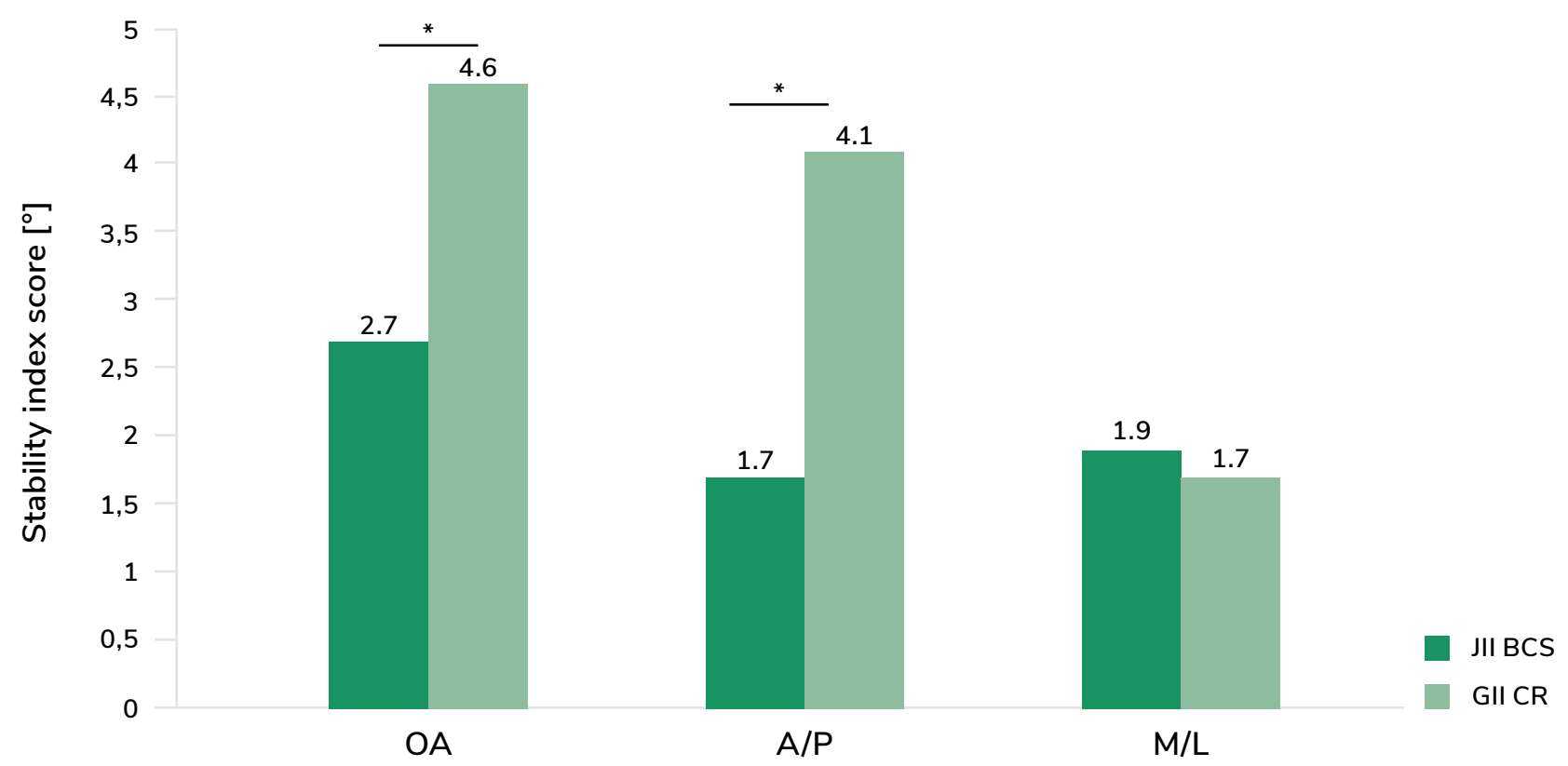

Figure 3. Mean results of single leg stability test for the operated extremities in Journey II BCS (JII BCS) and Genesis II CR groups (GII CR).

Abbreviations: JII BCS - Journey II Bi-cruciate Stabilized Total Knee System; GII CR - Genesis II Cruciate-Retaining; $\mathrm{OA}$ - overall index; A/P - anterior-posterior index; M/L - medial-lateral index; $(*)$ - statistically significant $(\mathrm{p}<0.05)$.

The JII BCS group obtained a WOMAC total score of $19.4 \pm 7.7$, whereas the GII CR group had a score of $38 \pm 13$. This identifiable difference was statistically significant $(\mathrm{p}=0.0026)$. We found smaller and non-significant differences in pain and stiffness, but all the scores were better in the JII BCS group. For physical function assessment, a statistically significant difference $(p=0.0009)$ was obtained between the JII BCS and GII CR prosthesis (12 \pm 4.4 vs $27 \pm 9.4$; Table 3$)$.

Table 3. Outcomes of the WOMAC questionnaire in Journey II BCS (JII BCS) and Genesis II CR groups (GII CR).

\begin{tabular}{|l|l|l|l|}
\hline Outcome & JII BCS & GII CR & P-value \\
\hline Pain & $4.3( \pm 2.3)$ & $7.4( \pm 4.3)$ & 0.072 \\
\hline Stiffness & $3.1( \pm 1.5)$ & $3.6( \pm 14)$ & 0.533 \\
\hline Physical Function & $12( \pm 4.4)$ & $27( \pm 9.4)$ & $0.0005^{\star}$ \\
\hline Total Score & $19.4( \pm 7.7)$ & $38( \pm 13)$ & $0.0002^{\star}$ \\
\hline
\end{tabular}

Notes: All values expressed as mean \pm standard deviation.

Abbreviations: JII BCS - Journey II Bi-cruciate Stabilized Total Knee System; GII CR - Genesis II Cruciate-Retaining; $(*)$ - statistically significant $(\mathrm{p}<0.05)$. 


\section{Discussion}

Our findings showed that performing TKA with bi-cruciate stabilized prostheses can positively influence early functional outcomes compared with TKA using the standard cruciate-retaining implants. The JII BCS group demonstrated superior, statistically significant differences in total AROM, mean difference in ATT between the operated and non-operated extremities, body balance in the SLBT, and clinical subjective score in the WOMAC questionnaire. The importance of both cruciate ligaments in restoring normal knee kinematics and suggestions to consider bi-cruciate implants as replacements for the function of native cruciate ligaments have been previously reported [21,22]. Using fluoroscopy, Grieco et al. [22] analyzed the in vivo kinematics of 50 knees during full weight-bearing range of motion. Of these knees, 40 received a JII BCS implant and 10 were normal asymptomatic knees. The authors concluded that the JII BCS implant exhibits normal-like kinematic patterns and moves in vivo as desired. Moreover, the kinematic patterns between the knees with a JII BCS implant and the asymptomatic knees suggest the dual post/cam design, and the asymmetrical articular geometries of the JII BCS adequately replicate ACL and PCL functions [22]. Iriuchishima et al. [31] examined the rollback of the JII BCS implant, compared this with a group of Oxford unicompartmental knees and a control group (non-operated extremity of the Oxford unicompartmental group), and found no inter-group differences in the rollback of the femur and ROM. The authors concluded that the JII BCS TKA design is likely to reproduce native ACL and PCL functions and native knee rollback. This suggests that restoration of full ROM after TKA could be considered a crucial factor for regaining adequate knee joint kinematics. In the present study, we did not observe any significant differences in flexion and extension AROM between the JII BCS and GII CR groups (mean flexion: $115.1^{\circ}$ vs $102.4^{\circ}$; mean extension deficit: $0.2^{\circ}$ vs $6.6^{\circ}$ ), but the total AROM in the JII BCS group was significantly better than that in the GII CR group $\left(114.9^{\circ}\right.$ vs $\left.95.9^{\circ}\right)$. On the basis of the presented short-term AROM results, we could attempt to predict satisfactory long-term outcomes after TKA with the JII BCS implant, as has already been reported by other authors [23,24].

In this study, knee stability was measured using two different devices, the GNRB arthrometer for measuring the ATT, which can be considered a "mechanical" stability, and the Biodex Balance System device to perform the SLBT as an indicator of lower extremity neuromuscular/balance control. To the best of our knowledge this is the first report to compare the JII BCS and GII CR implants by using arthrometry and balance platform devices. Inui et al. intraoperatively measured ATT in 62 patients after TKA with the JII BCS implant and 3 months postoperatively with a KT 2000 arthrometer at $30^{\circ}$ knee flexion. The intraoperative and postoperative ATT values were $7.7 \pm 3.1$ and $5.9 \pm 1.7$, respectively, with a positive correlation between these outcomes $(R=0.61)$ [25]. In the cruciate-retaining study, Niki et al. measured ATT with a KT 2000 device at 7.5 years postoperatively and obtained a result of $8.3 \pm 0.48$ $\mathrm{mm}$ [26]. Chouteau et al. [27] used a radiographic technique to quantify ATT during the anterior drawer test 3 months postoperatively and obtained an ATT of $4 \mathrm{~mm}$. We recorded a mean ATT of $6.2 \pm 1.3 \mathrm{~mm}$ for the JII BCS group and $8.3 \pm 3.5 \mathrm{~mm}$ for the GII CR group. The extremities implanted with the JII BCS showed (on average) an ATT of 2.1 $\mathrm{mm}$ less than that in the extremities implanted with the GII CR. Similar results were observed in the side-to-side comparison of the operated and non-operated knees. The patients with a GII CR prosthesis had $2.3 \mathrm{~mm}$ more ATT in the operated extremity, whereas the JII BCS group obtained a similar ATT between the extremities (difference of $0.2 \mathrm{~mm}$ ). Consequently, these outcomes show that patients with a JII BCS implant presented with more stable knees, with a minimum side-to-side difference in ATT. The shape of the slope of the curves and the side-to-side differences in the JII BCS group correspond with the data obtained from a healthy population [19]. 
Previous studies demonstrated that patients presented with impaired postural control [28], proprioception [29], and increased risk of falls after TKA [30]. Therefore, lower extremity balance control may be critical for the functional outcome and potential injury risk after TKA. The results of our study suggest that TKA with the JII BCS prosthesis is significantly better than GII CR in terms of single-leg balance, especially in $\mathrm{OA}$ $\left(2.7^{\circ} \pm 1.5^{\circ}\right.$ vs $\left.4.6^{\circ} \pm 1.4^{\circ}\right)$ and $\operatorname{AP}\left(1.7^{\circ} \pm 0.8^{\circ}\right.$ vs $4.1^{\circ} \pm$ $1.3^{\circ}$ ), with no significant difference in the ML balance index. Following the normative data of the balance indexes, the average outcome for healthy subjects (aged $54-71$ years) is $2.3^{\circ} \pm 1.4^{\circ}$. In other words, the healthy population outcome in this age group ranges from $0.9^{\circ}$ to $3.7^{\circ}[25]$.

\section{Limitations of the study}

This study is a preliminary report and limited by the number of patients evaluated. We did not have any knowledge concerning the subjects' functional state before the surgery. Surgical techniques and intraoperative soft tissue handling might have influenced our results (e.g., AROM).

\section{Conclusions}

The design of the JII BCS prosthesis may lead to better active ROM and could result in better joint kinematics (less paradoxical movement and more rollback). Restoration of the ACL and PCL func- tions with a dual post/cam system would lead to better mechanical stability of the knee joint (less AP tibial translation) and could result in better neuromuscular control of the lower extremity, as evaluated using the SLBT. The better functional outcome with the JII BCS Total Knee System could be considered a sum of all the registered clinical variables.

\section{Abbreviations}

$\mathrm{AP}$ - anteroposterior stability index;

AROM - active range of motion;

ATT - anterior tibial translation;

GII CR - Genesis II Cruciate-Retaining;

ICC - intraclass correlation coefficient;

ML - mediolateral stability index;

OA - overall stability index;

SLBT - single-leg balance test;

TKA - total knee arthroplasty;

WOMAC - Western Ontario and McMaster Universities Osteoarthritis.

\section{Acknowledgements}

The authors would like to thank to Magdalena Chłapek, Aleksandra Martyna and Kamil Kowalik for technical support. Additional information is that current workplace of the authors J.D. and A.P. is Klinika Nieborowice, Poland. 


\section{References}

1. Nilsdotter AK, Toksvig-Larsen S, Roos EM. Knee arthroplasty: are patients' expectations fulfilled? A prospective study of pain and function in $102 \mathrm{pa}^{-}$ tients with 5-year follow up. Acta Orthop. 2009; 80 (1): 55-61.

2. Argenson JN, Boisgard S, Parratte S, Descamps S, Bercovy M, Bonnevialle P. Survival analysis of total knee arthroplasty at a minimum 10 years' follow-up: a multicenter French nationwide study including 846 cases. Orthop Traumatol Surg Res. 2013; 99 (4): 385-390.

3. Scott CE, Howie CR, MacDonald D, Biant LC. Predicting dissatisfaction following total knee replacement: a prospective study of 1217 patients. J Bone Joint Surg Br. 2010; 92 (9): 1253-1258.

4. Bourne RB, Chesworth B, Davis A, Mahomed N, Charron K. Comparing patient outcomes after THA and TKA - is there a difference? Clin Orthop Relat Res. 2010; 468: 542-554.

5. Noble PC, Gordon MJ, Weiss JM, Reddix RN, Conditt MA, Mathis KB. Does total knee replacement restore normal knee function? Clin Orthop Relat Res. 2005; 431: 157-165.

6. Banks S, Bellemans J, Nozaki H, Whiteside LA, Harman M, Hodge WA. Knee motions during maximum flexion in fixed and mobile-bearing arthroplasties. Clin Orthop Relat Res. 2003; 410: 131-138.

7. Banks SA, Hodge WA. Design and activity dependence of kinematics in fixed and mobile-bearing knee arthroplasties. J Arthroplasty. 2004; 19 (7): 809-816.

8. Fantozzi S, Catani F, Ensini A, Leardini A, Giannini S. Femoral rollback of cruciate-retaining and posterior-stabilized total knee replacements: in vivo fluoroscopic analysis during activities of daily living. J Orthop Res. 2006; 24 (12): 2222-2229.

9. Pritchett JW. Patient preference in knee prostheses. J Bone Joint Surg Br. 2004; 86 (7): 979-982.

10. Murakami K, Hamai S, Okazaki K, Gondo H, Wang $\mathrm{Y}$, Ikebe S, et al. Knee kinematics in bi-cruciate stabilized total knee arthroplasty during squatting and stair-climbing activities. J Orthop 2018; 15 (2): 650-654.
11. Murakami K, Hamai S, Okazaki K, Wang Y, Ikebe S, Higaki $\mathrm{H}$, et al. In vivo kinematics of gait in posterior-stabilized and bicruciate-stabilized total knee arthroplasties using image-matching techniques. Int Orthop. 2018; 42 (11): 2573-2581.

12. Ries M. Effect of ACL sacrifice, retention, or substitution on kinematics after TKA. Orthopedics 2007; 30 (8 Suppl): 74-76.

13. Kuroyanagi Y, Mu S, Hamai S, Robb WJ, Banks SA. In vivo knee kinematics during stair and deep flexion activities in patients with bicruciate substituting total knee arthroplasty. J Arthroplasty 2012; 27 (1): 122-128.

14. Victor J, Mueller JK, Komistek R, Sharma A, Nadaud $\mathrm{M}$, Bellemans J. In vivo kinematics after a cruciate-substituting TKA. Clin Orthop Relat Res. 2010; 468 (3): 807-814.

15. Bade MJ, Kohrt WM, Stevens-Lapsley JE. Outcomes before and after total knee arthroplasty compared to healthy adults. J Orthop Sports Phys Ther. 2010; 40 (9): 559-567.

16. Rothstein JM, Miller PJ, Roettger RF. Goniometric reliability in a clinical setting. Elbow and knee measurements. Phys Ther. 1983; 63: 1611-1615.

17. Koo TK, Li MY. A guideline of selecting and reporting intraclass correlation coefficients for reliability research. J Chiropr Med. 2016; 15 (2): 155-163.

18. Robert H, Nouveau S, Gageot S, Gagnière B. A new knee arthrometer, the GNRB: experience in ACL complete and partial tears. Orthop Traumatol Surg Res. 2009; 95: 171-176.

19. Arnold BL, Schmitz RJ. Examination of balance measures produced by the Biodex Stability System. J Athl Train. 1998; 33 (4): 323-327.

20. Angst F, Aeschlimann A, Steiner W, Stucki G. Responsiveness of the WOMAC osteoarthritis index as compared with the SF-36 in patients with osteoarthritis of the legs undergoing a comprehensive rehabilitation intervention. Ann Rheum Dis. 2001; 60: 834-840.

21. Ward TR, Burns AW, Gillespie MJ, Scarvell JM, Smith PN. Bicruciate-stabilised total knee replacements produce more normal sagittal plane kinematics than posterior-stabilised designs. J Bone Joint Surg Br. 2011; 93 (7): 907-913. 
22. Grieco TF, Sharma A, Dessinger GM, Cates HE, Komistek RD. In vivo kinematic comparison of a bicruciate stabilized total knee arthroplasty and the normal knee using fluoroscopy. J Arthroplasty 2018; 33 (2): 565-571.

23. Iriuchishima T, Ryu K. A comparison of rollback ratio between bicruciate substituting total knee arthroplasty and Oxford unicompartmental knee arthroplasty. J Knee Surg. 2018; 31 (6): 568-572.

24. Hommel H, Wilke K. Good early results obtained with a guided-motion implant for total knee arthroplasty: a consecutive case series. Open Orthop J. 2017; 11: 51-56.

25. Inui H, Taketomi S, Yamagami R, Kawaguchi K, Nakazato K, Tanaka S. The relationship between anteroposterior stability and medial-lateral stability of the bi-cruciate stabilized total knee arthroplasty. Knee 2018; 25 (6): 1247-1253.

26. Niki Y, Mochizuki T, Momohara S, Saito S, Matsumoto H, Tomatsu T. Factors affecting anteroposterior instability following cruciate-retaining total knee arthroplasty in patients with rheumatoid arthritis. Knee 2008; 15 (1): 26-30.
27. Chouteau J, Lerat JL, Testa R, Moyen B, Banks SA. Sagittal laxity after posterior cruciate ligament-retaining mobile-bearing total knee arthroplasty. J Arthroplasty 2009; 24 (5): 710-715.

28. Gage WH, Frank JS, Prentice SD, Stevenson P. Postural responses following a rotational support surface perturbation, following knee joint replacement: frontal plane rotations. Gait Posture 2008; 27 (2): 286-293.

29. Fuchs S, Thorwesten L, Niewerth S. Proprioceptive function in knees with and without total knee arthroplasty. Am J Phys Med Rehabil. 1999; 78: 39-45.

30. Swinkels A. Newman JH. Allain TJ. A prospective observational study of falling before and after knee replacement surgery. Age Ageing 2009; 78 (1): 175181. 